Abellán-Rubio, J., Arnaiz-Sánchez, P. \& Alcaraz-García, S. (2021). El profesorado de apoyo y las barreras que interfieren en la creación de apoyos educativos inclusivos. Revista Electrónica Interuniversitaria de Formación del Profesorado, 24(3), 237-249.

DOI: https://doi.org/10.6018/reifop.486901

\title{
El profesorado de apoyo y las barreras que interfieren en la creación de apoyos educativos inclusivos
}

Javier Abellán Rubio, Pilar Arnaiz Sánchez, Salvador Alcaraz García

Universidad de Murcia

\section{Resumen}

La consecución de apoyos educativos inclusivos precisa de una respuesta a la diversidad común para todo el centro, que genere espacios que aseguren la presencia, la participación y el logro socioeducativo de todos los estudiantes. El objetivo general de este estudio es analizar cómo se desarrolla el apoyo educativo desde el marco de acción de la educación inclusiva en una comarca de la Región de Murcia. Para ello, se llevó a cabo una investigación descriptiva, no experimental y de corte mixto, a través de la utilización de un cuestionario en el que participaron 23 docentes de apoyo. Los resultados evidencian una desvinculación en las tareas del profesorado de apoyo hacia el aula ordinaria, una atención, en gran medida, individualizada y fuera del aula ordinaria hacia el alumnado que recibe apoyo, y una falta de coordinación y trabajo conjunto por parte de los docentes de apoyo y los docentes de aula ordinaria. Se concluye que la transformación de las barreras que dificultan el asentamiento de sistemas de apoyo inclusivo en facilitadores para la inclusión, pueden venir determinadas, por la existencia de un currículo más flexible, la formación docente en inclusión y la reformulación de los roles docentes hacia perfiles más colaborativos.

Palabras clave

Educación inclusiva; modelo de apoyo; barreras en el apoyo educativo; profesorado de apoyo.

\section{Contacto:}

Pilar Arnaiz Sánchez, parnaiz@um.es, Facultad de Educación, Campus de Espinardo, 30100, Murcia, España. 


\title{
Support teachers and the barriers that interfere with the creation of inclusive educational supports
}

\begin{abstract}
The attainment of inclusive educational support requires a common response to diversity for the whole centre, which generates spaces that ensure the presence, participation and socioeducational achievement of all students. The general objective of this study is to analyse how educational support is developed from the framework of action of inclusive education in a territory from the Region of Murcia. For this purpose, a descriptive, non-experimental and mixed research was carried out using a questionnaire in which 23 support teachers participated. The results show a dissociation in the tasks of the support teachers towards the regular classroom, a largely individualised attention outside the regular classroom towards the students who receive support, and a lack of coordination and joint work on the part of the support teachers and the regular classroom teachers. It is concluded that the transformation of the barriers that hinder the establishment of inclusive support systems into facilitators for inclusion, can be determined by the existence of a more flexible curriculum, teacher training in inclusion and the reformulation of teaching roles towards more collaborative profiles.
\end{abstract}

\section{Key words}

Inclusive education; support model; barriers in educational support; support teachers.

\section{Introducción}

Desde el marco de acción de la educación inclusiva los apoyos son toda estrategia, material, recurso o actuación que se lleva a cabo con la intencionalidad de lograr el acceso, la participación y el aprendizaje de sus destinatarios en el contexto educativo (Arnaiz, 2019; Hagiwara, et al., 2019). Booth y Ainscow (2015) los definen como aquellas "actividades que aumentan la capacidad de un centro escolar para atender a la diversidad del alumnado promoviendo que todo el mundo sea valorado con equidad" (p. 50). Estas definiciones enfatizan la comprensión del apoyo como un recurso común para todo el centro, a través de la creación de procesos de enseñanza-aprendizaje inclusivos acordes a las verdaderas realidades del aula ordinaria (Arnaiz y De Haro, 2020; Härkki, Vartiainen, SeitamaaHakkarainen y Hakkarainen, 2021).

En España existen dos figuras de apoyo esenciales: el profesorado de Pedagogía Terapéutica (PT) y el de Audición y Lenguaje (AL). Sus funciones, aunque quedan establecidas y reguladas en la legislación, en la práctica varían dependiendo del contexto, lo que conlleva a veces una falta de claridad en sus labores (Moya, 2012). En ocasiones se genera en torno a estos docentes una visión del apoyo restrictiva y balcanizada, tras la que se crean espacios, roles y tiempos profesionales diferenciados e independientes del aula regular, lo que produce que el apoyo recaiga solamente en ellos y no se comparta con toda la comunidad educativa.

Entre las funciones principales del profesorado de apoyo se encuentra la atención individualizada o en pequeños grupos al alumnado con necesidades de apoyo educativo, actuaciones que mayoritariamente se realizan en aulas especializadas (Sanahuja, Mas y 
Olmos, 2018; Sandoval, Márquez, Simón y Echeita, 2019; Simón, Echeita y Sandoval, 2018). Este hecho reduce las potencialidades que los apoyos pueden aportar a los centros educativos, y suponen una de las principales razones de segregación y exclusión educativa para determinados alumnos, habida cuenta que perjudican sus posibilidades de diálogo, interacción y socialización con sus iguales y con el docente tutor (Ní Bhroin y King, 2020).

Los apoyos deberían llevarse a cabo en el aula ordinaria, lugar de referencia y de aprendizaje para todos los estudiantes (Tsvintarnaia et al., 2020). Por ello, algunos autores destacan la descontextualización que se produce entre el profesorado de apoyo y el de aula ordinaria, debido a la falta de coordinación, comunicación y planificación conjunta (Luque et al., 2018). Anaby, lanni, Héguy y Camden (2020) expresan que para la creación de apoyos inclusivos no son suficientes la coordinación y el diálogo entre el profesorado regular y el de apoyo. Se precisa, además, una redefinición de ambos roles que genere transformaciones ideológicas y funcionales en las labores de apoyo, para que atender a la diversidad sea una tarea compartida en los centros. En palabras de Quintero (2020), este cambio se ha de proyectar a través de una adecuada formación -inicial y permanente- que asegure la creación de profesionales que actúen como "agentes para la inclusión".

Sanahuja et al. (2018) justifican la descoordinación de ambos perfiles a causa de la falta de formación en diversidad que presentan los docentes de las aulas regulares. Ello produce que deleguen en el profesorado de apoyo la responsabilidad de la atención del alumnado con necesidad de apoyo educativo (Arnaiz, Escarbajal, Alcaraz y de Haro, 2021). Por consiguiente, la redefinición de los roles docentes constituye una premisa fundamental para crear cambios metodológicos que favorezcan la inclusión de todo el alumnado en las aulas ordinarias.

En cuanto a la coordinación y el diálogo entre el profesorado, Nadal, Grau y Peirats (2016) comprobaron que el perfil docente parece influir en dicha coordinación, ya que el profesorado de PT se encuentra más vinculado al aula ordinaria en su desempeño profesional que el profesorado de AL. Incluso obtuvieron evidencias de que cuando el profesorado de apoyo establece su labor dentro del aula ordinaria, ambos docentes llevan a cabo funciones paralelas. A este respecto, Sandoval, Simón y Echeita (2012) indican que es común encontrar que en estas situaciones de trabajo conjunto sea el tutor el que dirija la clase, mientras que el docente de apoyo se encarga de la atención individualizada del alumnado con necesidades educativas especiales. Esta diferenciación en las tareas afecta al alumnado que recibe apoyo y al propio docente que lo imparte, siendo considerado como un docente con unas responsabilidades delimitadas tanto en el aula como en el centro.

El docente de apoyo expresa su excesiva carga de trabajo (Anaby et al., 2020) y su falta de tiempo para atender al alumnado que lo requiere. Esta realidad dificulta la construcción de procesos educativos conjuntos a través de la denominada docencia compartida o coenseñanza entre todos los profesionales como expresa Dagli, Akcamete y Guneyli (2020).

El profesorado de apoyo no representa, pues, en España -como en otros países- la figura de un coordinador o asesor para la inclusión (Ainscow, 2017), cuyas labores se vinculan hacia la orientación e información al docente de aula ordinaria, la creación de materiales específicos y el diseño de actividades para el alumnado que recibe apoyo, sino que se ocupa de la docencia y del apoyo directo al alumnado (Gómez-Zepeda, Petreñas, Sabando y Puigdellivol, 2017; Soldevilla, Naranjo y Muntaner, 2017). Por tanto, los centros deberían organizar y generar la flexibilidad de espacios, tiempos y roles que requiere la educación inclusiva, para adaptarse, per se, a los diferentes ritmos, necesidades, intereses y características de su alumnado (Sanahuja, Moliner y Moliner, 2020). 
En relación a las consideraciones indicadas, el objetivo general de este estudio es analizar cómo se desarrolla el apoyo educativo desde el marco de acción de la educación inclusiva en los centros educativos de una comarca de la Región de Murcia.

Los objetivos específicos derivados del mismo son:

1. Conocer las funciones del profesorado de apoyo en su desempeño profesional.

2. Detectar las barreras que obstaculizan la implementación de apoyos educativos inclusivos.

3. Identificar las actuaciones que favorecerían la creación de apoyos inclusivos en los centros educativos.

\section{Metodología}

\subsection{Diseño de la investigación}

La finalidad de este estudio es analizar y describir los fenómenos que ocurren en los centros educativos a nivel de resultados. Por tanto, se enmarca dentro de un diseño descriptivo, no experimental y de corte mixto, puesto que la información que se obtiene para dar respuesta a los objetivos marcados se representa a través de datos cuantitativos y cualitativos.

\subsection{Participantes}

Esta investigación se ha desarrollado en una comarca de la Región de Murcia, que cuenta con ocho centros educativos de infantil, primaria y secundaria, con una población de 28 docentes de apoyo (PT y/o AL). La muestra real fue de 23 participantes (Tabla 1), lo que supone un tamaño muestral con un nivel de confianza del $95 \%(Z=1,96)$.

\section{Tabla 1.}

Características sociodemográficas de la muestra participante

\begin{tabular}{rc}
\hline & $\mathbf{N}(\%)$ \\
\hline Etapa Educativa \\
\hline Infantil y Primaria & $16(69.6 \%)$ \\
\hline Secundaria & $7(30.4 \%)$ \\
\hline Perfil Profesional \\
\hline PT & $14(60.9 \%)$ \\
AL & $5(21.7 \%)$ \\
\hline PT/AL & $4(17.4 \%)$ \\
\hline Experiencia docente \\
\hline $0-5$ años: & $5(21.7 \%)$ \\
\hline 6-10 años & $5(21.7 \%)$ \\
\hline Más de 10 años & $13(52.2 \%)$ \\
\hline TOTAL & $23(100 \%)$ \\
\hline
\end{tabular}

\subsection{Instrumento}

El instrumento de recogida de información fue el Cuestionario sobre las funciones del profesorado de apoyo (Sandoval et al., 2019). En la Tabla 2 se expone la estructura del cuestionario, conformado por cuatro dimensiones y 28 ítems. 
Tabla 2.

Estructura del cuestionario: dimensiones, ítems y tipología de las preguntas

\begin{tabular}{|c|c|c|c|}
\hline DIMENSIONES & ÍTEMS & TIPOLOGÍA & $\begin{array}{c}\text { ALFA DE } \\
\text { CRONBACH }\end{array}$ \\
\hline \multirow{5}{*}{ Perfil profesional } & Género & \multirow{5}{*}{$\begin{array}{l}\text { Cerradas } \\
\text { elección múltiple }\end{array}$} & \\
\hline & Puesto laboral & & \\
\hline & Antigüedad docente & & \\
\hline & Etapa educativa & & \\
\hline & $\begin{array}{l}\text { Titularidad del centro o tipo de } \\
\text { centro }\end{array}$ & & \\
\hline \multirow{7}{*}{$\begin{array}{c}\text { Funciones } \\
\text { desarrolladas }\end{array}$} & $\begin{array}{l}\text { Ítem 1. Atención } \\
\text { individual/grupal fuera del aula }\end{array}$ & \multirow{7}{*}{ Likert 1 a 3} & \multirow{7}{*}{.710} \\
\hline & Ítem 2. Atención en el aula & & \\
\hline & $\begin{array}{l}\text { Ítem 3. Co-docencia al grupo } \\
\text { clase }\end{array}$ & & \\
\hline & $\begin{array}{l}\text { Ítem 4. Elaboraciones materiales } \\
\text { de apoyo }\end{array}$ & & \\
\hline & $\begin{array}{l}\text { Ítem } 5 . \text { Información a docentes } \\
\text { sobre apoyo }\end{array}$ & & \\
\hline & $\begin{array}{l}\text { Ítem 6. Coordinación planes de } \\
\text { trabajo individual }\end{array}$ & & \\
\hline & $\begin{array}{l}\text { Ítem 7. Sustitución compañeros } \\
\text { de baja }\end{array}$ & & \\
\hline \multirow{14}{*}{$\begin{array}{l}\text { Barreras y } \\
\text { limitaciones }\end{array}$} & $\begin{array}{l}\text { Ítem } 8 \text {. Actitudes negativas hacia } \\
\text { la inclusión }\end{array}$ & \multirow{14}{*}{ Likert 1 a 3} & \multirow{14}{*}{.913} \\
\hline & $\begin{array}{l}\text { Ítem 9. Escasa cooperación del } \\
\text { profesorado }\end{array}$ & & \\
\hline & $\begin{array}{l}\text { Ítem } 10 . \text { Aumento de la carga de } \\
\text { trabajo }\end{array}$ & & \\
\hline & Ítem 11. Cambios en la plantilla & & \\
\hline & $\begin{array}{l}\text { Ítem } 12 . \text { Escasa flexibilidad del } \\
\text { profesorado }\end{array}$ & & \\
\hline & $\begin{array}{l}\text { Ítem } 13 . \text { Falta de apoyo del } \\
\text { equipo directivo }\end{array}$ & & \\
\hline & $\begin{array}{l}\text { Ítem } 14 . \text { Falta de apoyo del } \\
\text { orientador/a }\end{array}$ & & \\
\hline & $\begin{array}{l}\text { Ítem } 15 . \text { Estrategias y } \\
\text { metodologías rígidas }\end{array}$ & & \\
\hline & Ítem 16. Falta de visión conjunta & & \\
\hline & $\begin{array}{l}\text { Ítem 17. Escaso tiempo y } \\
\text { espacios de coordinación }\end{array}$ & & \\
\hline & $\begin{array}{l}\text { Ítem 18. Reticencia a compartir } \\
\text { aula }\end{array}$ & & \\
\hline & $\begin{array}{l}\text { Ítem 19. Identificación de las } \\
\text { funciones de apoyo }\end{array}$ & & \\
\hline & $\begin{array}{l}\text { Ítem 20. Profesorado apoyo de } \\
\text { categoría inferior }\end{array}$ & & \\
\hline & $\begin{array}{l}\text { Ítem 21. Falta de apoyo de la } \\
\text { administración }\end{array}$ & & \\
\hline
\end{tabular}




\begin{tabular}{clc}
\hline & $\begin{array}{l}\text { Ítem 22. Falta de implicación } \\
\text { inspección }\end{array}$ & Abierta \\
\hline $\begin{array}{c}\text { Elementos que } \\
\text { favorecerían la } \\
\text { inclusión }\end{array}$ & $\begin{array}{l}\text { Ítem 23. Funciones que } \\
\text { favorecerían la inclusión }\end{array}$ \\
\hline & Fuente: Elaboración propia, a partir de Sandoval et al. (2019).
\end{tabular}

\subsection{Procedimiento y análisis de datos}

Se realizó una revisión bibliográfica para analizar la temática objeto de estudio. Seleccionada la comarca, se accedió a los centros que la componen, informándoles de la investigación y solicitando su participación. Tras el estudio empírico, se realizó un nuevo encuentro con los centros donde se aportó los datos del estudio y posibles actuaciones de mejora.

Para el análisis cuantitativo se procedió a calcular los estadísticos descriptivos para cada uno de los ítems del cuestionario (medias y frecuencias).

Para el análisis inferencial, se aplicó la prueba Shapiro-Wilk que demostró la distribución no normal de los datos ( $\mathrm{p}$ <.001). Como variables predictoras se utilizó el puesto laboral (PT, AL y PT/AL) y la experiencia docente (0-5 años, 6 a 10 años o más de 10 años). Para su análisis se aplicó la prueba estadística no paramétrica $U$ de Mann-Whitney y la prueba Chi-Cuadrado de Pearson, estableciendo un valor de significancia $p \leq .005$. Estos análisis se realizaron con el paquete estadístico SPSS (versión 25 para Windows).

Para los datos cualitativos se realizó un análisis de contenido de las respuestas del ítem 23. Se siguió un modelo inductivo, estableciéndose las categorías de análisis y los códigos para la clasificación de la información. Estos análisis se realizaron mediante el programa Atlas.Ti (versión 8 para Windows).

\section{Resultados}

Respecto al objetivo 1, describir las funciones que realiza el profesorado de apoyo en su labor docente, los resultados indican que la función que con mayor frecuencia realiza el profesorado de apoyo es la elaboración de fichas y de material específico para el alumnado que recibe el apoyo $\left(M_{\text {item }}=2.96\right)$. El total de participantes realizan esta función $(4.3 \%$ esporádicamente y $95.7 \%$ con mucha frecuencia).

Otras de las funciones que en mayor medida realizan los profesionales de apoyo participantes es la atención educativa, individual o en pequeño grupo, fuera del aula ordinaria ( $M_{\text {item1 }}=2.91$ ), y la información a los compañeros del centro sobre temas relacionados con el alumnado que precisa apoyo ( $\left.M_{\text {ítem5 }}=2.91\right)$. En ambos casos, el $91.3 \%$ del profesorado señala que realiza con mucha frecuencia dichas labores, siendo un $8.7 \%$ el que las lleva a cabo esporádicamente. De igual forma, la coordinación entre docentes, para planificar y hacer seguimiento de los planes de trabajo individuales (Mítem6 $=2.83$ ), ocupa un alto porcentaje del tiempo del profesorado de apoyo. En este sentido, el $82.6 \%$ del profesorado alega coordinarse muy a menudo con sus compañeros de aula ordinaria, mientras que un $17.4 \%$ señala que lo hace de manera esporádica.

Con menor frecuencia realizan la atención educativa con el alumnado que recibe apoyo dentro del aula ordinaria $\left(M_{\text {item2 }}=2.43\right)$. Tan solo el $47.8 \%$ de los encuestados declara intervenir con el alumnado dentro de su aula ordinaria con mucha frecuencia. El $47.8 \%$ realiza esta función esporádicamente. 
Entre las funciones que tienen menor frecuencia en la jornada del profesorado de apoyo se encuentran: la sustitución a compañeros por bajas o incidencias escolares $\left(M_{\text {item7 }}=2.22\right)$, pues solo el $26.1 \%$ afirma realizarla frecuentemente; y la atención educativa al conjunto de un grupo clase junto con el docente de aula ordinaria $\left(M_{\text {item3 }}=1.70\right)$. Esta es la función menos destacada, puesto que un $52.2 \%$ del profesorado señala no realizar nunca esta función.

No se han hallado diferencias estadísticamente significativas de los resultados en función del perfil del profesorado de apoyo y su experiencia docente.

En relación al objetivo 2, detectar las barreras que obstaculizan la implementación de un modelo de apoyo inclusivo, en la Figura 1 se muestran las percepciones del profesorado sobre las barreras que, con un mayor grado de incidencia, dificultan el asentamiento de un sistema de apoyo verdaderamente inclusivo.

\section{Figura 1.}

Frecuencias de los factores que dificultan la labor de los especialistas en los apoyos

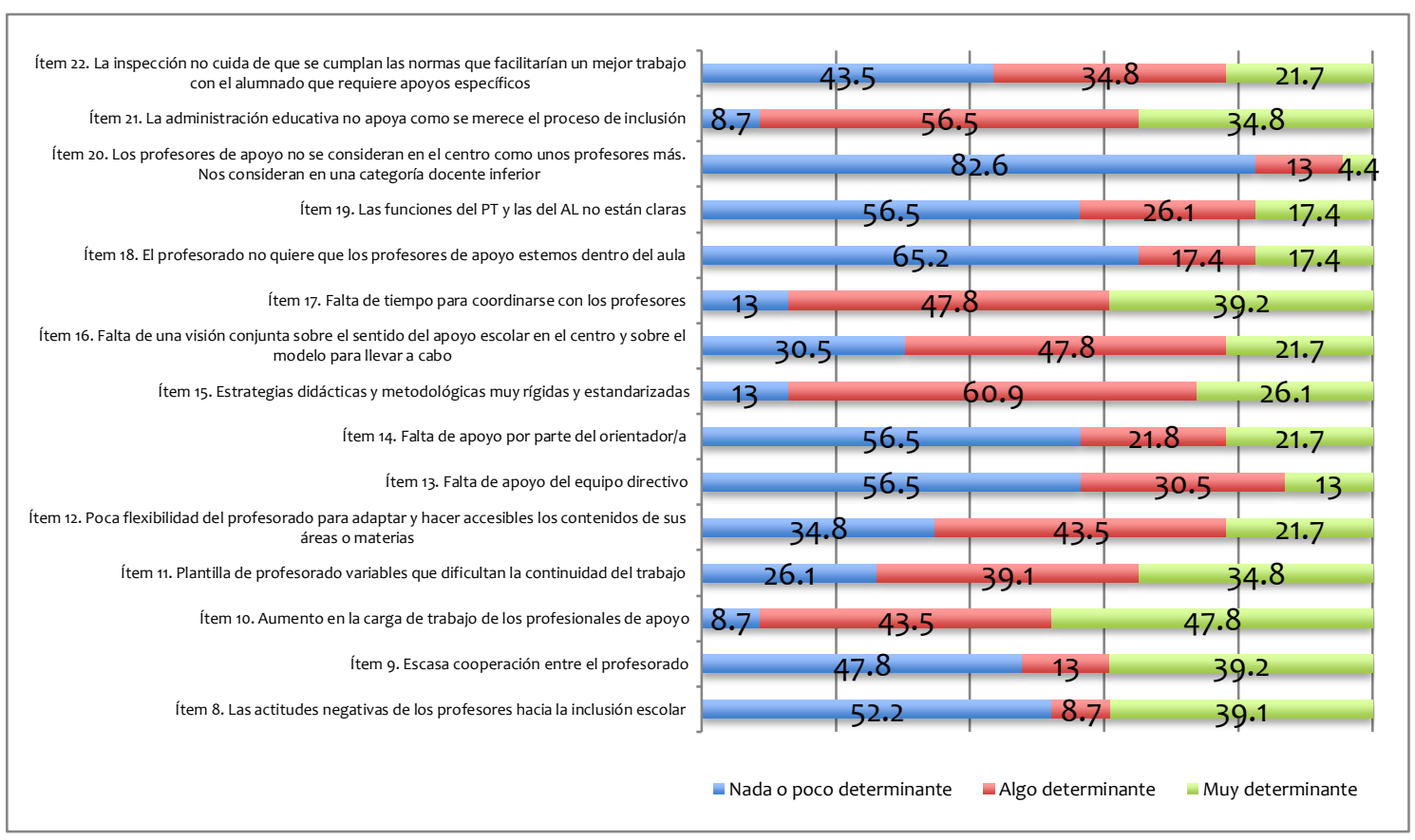

Fuente: Elaboración propia

Como se puede apreciar, el profesorado de apoyo destaca como principal barrera la gran carga de trabajo que asume en sus centros ( $M_{\text {item10 }}=2.39$ ), tan solo el $8.7 \%$ señala que esta variable es poco o nada determinante. Esto ocasiona falta de tiempo para coordinarse con los compañeros ( $M_{\text {itemi7 }}=2.26$ ), aunque el $13 \%$ no considere que la coordinación con el profesorado sea un factor que dificulte el trabajo de apoyo escolar. De igual modo, el $91.3 \%$ subraya la problemática que supone el escaso amparo que recibe el proceso de inclusión por parte de la administración educativa $\left(M_{\text {item21 }}=2.26\right)$. El $87 \%$ del profesorado expresa el planteamiento de estrategias didácticas y metodologías demasiado rígidas y estandarizadas como otro elemento que ha de reformularse $\left(M_{\text {item15 }}=2.13\right)$. $Y$ un $73.9 \%$ ha indicado que la variabilidad de la plantilla de docentes supone una dificultad en la consecución de apoyos inclusivos ( Mitem11 $_{\text {2.09). }}$. 
En menor medida, se destacan otros factores. Por ejemplo, el $52.1 \%$ del profesorado señala la escasa cooperación entre el profesorado $\left(M_{\text {itemg }}=1.91\right)$ y el $69.5 \%$ la falta de una visión conjunta sobre el modelo de apoyo que se debe llevar a cabo en el centro ( $M_{\text {item16 }}=1.91$ ). Además, el $65.2 \%$ indica la escasa flexibilidad del profesorado para adecuar los contenidos hacia las necesidades del alumnado que presenta mayores dificultades de aprendizaje ( $M_{\text {item12 }}=1.87$ ). De igual modo, el $65.2 \%$ enfatiza la existencia de actitudes negativas de los profesores hacia la inclusión escolar $\left(M_{\text {item8 }}=1.87\right.$ ) y el $56.5 \%$ alude al hecho de que la inspección no cuide el cumplimiento de la normativa, lo que influiría en la mejora de los apoyos educativos ( $M_{\text {ítem22 }}=1.78$ ).

Entre los elementos que menos dificultan la consecución de apoyos eficaces, desde el punto de vista inclusivo, el $17.3 \%$ indica la consideración al profesor de apoyo como un docente de una categoría inferior $\left(M_{\text {item2o }}=1.22\right)$, el $34.8 \%$ señala la negación del profesorado de aula a que el profesor de apoyo realice su labor dentro del aula ordinaria ( $\left.M_{\text {item18 }}=1.52\right)$, el $43.4 \%$ incide en la falta de apoyo por parte del equipo directivo ( $M_{\text {item13 }}=1.57$ ), el $43.5 \%$ indica la falta de claridad en las funciones que debe realizar el profesorado de PT y $A L\left(M_{\text {item19 }}=1.61\right)$ y el $43.4 \%$ destaca la falta de apoyo por parte del orientador ( $\left.M_{i \text { tem14 }}=1.65\right)$.

Con respecto al análisis de las diferencias estadísticamente significativas, los resultados corroboran que existen diferencias en el ítem: las funciones del PT y las del AL no están claras, atendiendo al puesto laboral que presenta el participante (chi-cuadrado $=9.322 ; \mathrm{gl}=2 ; p=$ .009). Estas diferencias se dan entre el AL y ambos perfiles ( $U=40.05 ; p=.016)$, así como entre el PT y ambos perfiles $(U=9.000 ; p=.017)$.

Atendiendo a la falta de una visión conjunta sobre el modelo de apoyo de centro, se han encontrado diferencias estadísticamente significativas en función de la experiencia docente (chi-cuadrado $=6.571 ; \mathrm{gl}=2 ; p=.037$ ). Estas diferencias se dan entre los docentes entre $6 \mathrm{y}$ 10 años de experiencia y los de o y 5 años $(U=37.000 ; p=.036)$.

Los resultados corroboran que existen diferencias en el ítem: el profesorado de aula no quiere que los docentes de apoyo estemos dentro del aula, con respecto a la experiencia docente de los participantes (chi-cuadrado $=6.646 ; \mathrm{gl}=2 ; p=.036$ ). Estas diferencias se dan entre el profesorado con experiencia superior a 10 años y el profesorado entre 6 y 10 años de experiencia $(U=36.000 ; p=.005)$.

En relación con el objetivo 3, identificar las actuaciones que favorecerían el proceso de inclusión en los centros educativos, los resultados muestran el amplio número de acciones que favorecerían apoyos educativos inclusivos en las escuelas.

De las respuestas aportadas por el profesorado se ha creado una red de códigos (Figura 2) que correlaciona los elementos que obstaculizan o, en su caso, facilitan el asentamiento de la inclusión en las escuelas.

De manera unánime, el profesorado de apoyo ha insistido en la necesidad de derribar las barreras que impiden la presencia, la participación y el aprendizaje del alumnado. La excesiva rigidez del currículo no permite el pleno desarrollo social y competencial del alumnado, lo que desemboca en estrategias metodológicas y roles docentes unilaterales y fuertemente estigmatizados. Por tanto, el profesorado destaca la necesidad de que en el aula ordinaria se lleven a cabo estrategias que potencien la transformación de los roles docentes y la flexibilidad del currículo educativo. Para ello considera prioritario la formación docente en materia de diversidad e inclusión si se quieren generar escuelas inclusivas. Mejoraría la coordinación y el diálogo docente -a causa de la responsabilidad compartida del alumnado-y 
potenciaría la inclusión de todo el alumnado en las aulas ordinarias a través de la cotutorización.

\section{Figura 2.}

Red de códigos sobre acciones que incentivarían la inclusión

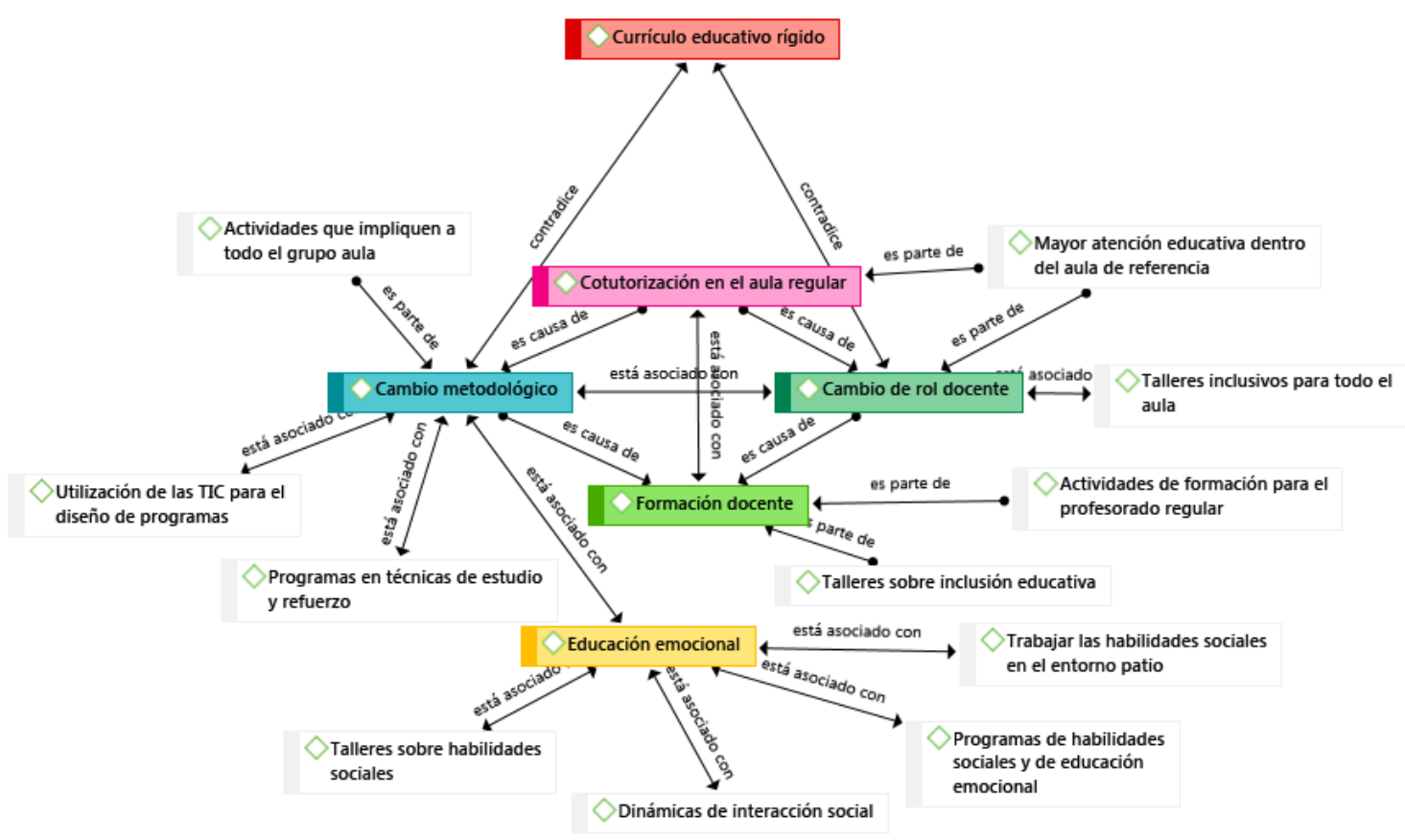

Fuente: Elaboración propia.

Y todo ello desde un clima de centro inclusivo y democrático, donde la creación de experiencias comunitarias y participativas, el desarrollo de la educación emocional y las habilidades sociales, incentiven la preparación de un sistema basado en valores humanos, sociales, democráticos y, por ende, inclusivos. Como indican Cortés, González y Sánchez (2018), el aula es un lugar de interacción donde la metodología, la organización y la manera de comprender y atender a la diversidad determinan las relaciones sociales y educativas generadas entre sus discentes, lo que favorece la atención a la diversidad y la inclusión.

\section{Discusión}

Este estudio ratifica dos labores principales que el profesorado de apoyo realiza: la creación de fichas y material específico, y la información a sus compañeros de aula sobre el alumnado que recibe apoyo, como ya revelaron otros estudios (Gómez-Zepeda et al., 2017; Soldevilla et al., 2017).

Otra de las funciones que el docente de apoyo destaca es la atención educativa individual o en pequeños grupos fuera del aula ordinaria. Estos datos se vinculan con recientes estudios (Sanahuja et al., 2018; Sandoval et al., 2019; Simón et al., 2018), que muestran la descontextualización entre el apoyo educativo y el aula ordinaria. Esto perpetúa la exclusión socioeducativa que sufre el alumnado en los procesos de enseñanza-aprendizaje generales, así como en las relaciones socioafectivas con sus iguales (Ní Bhroin y King, 2020). 
La mitad de los encuestados ha alegado que realiza el apoyo dentro del aula regular, mientras que para la otra mitad esta no es una labor frecuentada. A este respecto Sandoval et al. (2012) afirman que trabajar en el mismo aula no conlleva, per se, la existencia de situaciones de aprendizaje conjunto, ya que ambas funciones pueden realizarse de manera paralela.

El estudio no ha revelado diferencias significativas en la descontextualización del aula ordinaria atendiendo a los perfiles profesionales de apoyo existentes. Esta sí sucedía en el estudio de Nadal et al. (2016), donde el profesorado de PT tenía una vinculación más estrecha con el aula ordinaria que el docente de AL.

La mayoría del profesorado de apoyo ha destacado la coordinación como tarea prioritaria en su desempeño docente, pero un número significativo de ellos no contempla la colaboración como una función diaria. Estos resultados, aunque favorables, arrojan luz sobre la incógnita de la desvinculación existente entre el profesorado regular y el profesorado de apoyo, como ya lo hicieron otros estudios (Luque et al., 2018). Estos resultados parecen explicar el bajo índice de docentes que desarrolla el apoyo al conjunto de un grupo clase junto con el profesor regular y las casi inexistentes situaciones de co-enseñanza que se dan en los centros educativos estudiados (Dagli et al., 2020).

La mayor barrera que obstaculizan la creación de apoyos inclusivos, según los docentes de apoyo, es la excesiva carga de trabajo que asumen en sus centros (Anaby et al., 2020). Este hecho complica la generación de espacios inclusivos, ya que se ven debilitadas las relaciones docentes, dando lugar a otra de las barreras más indicadas por los especialistas: la falta de tiempo para coordinarse.

Este estudio ha mostrado, como ya lo hicieron otros (Sandoval et al., 2019; Takala et al., 2009), el escaso respaldo que recibe la inclusión por parte de la administración educativa. Hecho que alude a la necesidad de generar un nuevo impulso legislativo que guíe y proyecte la creación de contextos inclusivos.

Los docentes dan gran importancia a la realización de estrategias didácticas demasiado rígidas y estandarizadas como obstáculo para la construcción de apoyos inclusivos, así como la escasa flexibilidad del docente de aula para adecuar los contenidos hacia el alumnado que presenta mayores dificultades. Estos resultados corroboran los de Sanahuja et al. (2020), cuando inciden en la inexorable organización que requieren los centros educativos en sus tiempos y espacios para llegar a adecuarse a las diferentes necesidades, características y ritmos de sus educandos.

El profesorado de este estudio alude a la escasa cooperación entre los docentes, lo que constituye otra barrera para la eficacia de los apoyos educativos (Biedma, 2017) y repercute en la falta de una visión conjunta sobre el modelo de apoyo que se ha de desarrollar en los centros encuestados (Sandoval et al., 2019; Takala et al., 2009). Estos resultados muestran un inadecuado aprovechamiento del apoyo en los centros educativos, ya que este debería valorarse como un recurso general de centro para favorecer la diversidad y la inclusión (Härkki et al., 2021).

A diferencia del estudio desarrollado por Sanahuja et al. (2018), los docentes de la presente investigación no consideran que sus responsabilidades se encuentren delimitadas o que su función dentro de la escuela se vea relegada a una categoría inferior.

Otra de las barreras que el estudio ha refutado es la falta de claridad entre las funciones que han de desempeñar los especialistas de apoyo, datos que se contradicen con lo expuesto por Moya (2012). Sin embargo, esta barrera sí afecta al profesorado cuyo perfil profesional abarca el rol de PT y AL de forma simultánea, ya que estos últimos alegan que sus labores en el centro no se encuentran clarificadas. 
Además, el profesorado especialista reconoce que el docente de aula no supone un impedimento para la realización de apoyos dentro del aula ordinaria. No obstante, estas valoraciones no son compartidas por todos los profesionales de apoyo, ya que los que poseen una experiencia docente entre cero y cinco años consideran que el profesorado regular no quiere que el apoyo se efectúe dentro del este aula.

Los docentes han subrayado diversas actuaciones que, llevadas a cabo desde sus centros, favorecerían la creación de apoyos inclusivos. Estas acciones se entrelazan con la eliminación de las barreras que los docentes han señalado en el objetivo 2.

Los especialistas han insistido en posicionar el aula ordinaria como lugar de referencia donde desarrollar los aprendizajes, a través de estrategias metodológicas compartidas que impliquen a todos los estudiantes, a través de la co-enseñanza (Tsvintarnaia et al., 2020). Además, han expuesto la importancia de crear talleres, programas y proyectos inclusivos en los centros, de manera que fomenten la formación docente en diversidad para lograr un cambio en los roles a favor de la inclusión (Quintero, 2020). Todo ello motivado por un currículo educativo que rija la inclusión como principio (Arnaiz, 2019).

Por otra parte, el profesorado ha reconocido la importancia de la educación emocional del alumnado en pos de mejorar las relaciones sociales que se dan en la escuela, en beneficio de la diversidad (Cortés et al., 2018).

\section{Conclusiones}

Este estudio ha mostrado que el apoyo sigue inmerso en postulados propios del modelo del déficit donde este es entendido como un recurso terapéutico de atención directa al alumnado con mayores dificultades de aprendizaje, para paliar sus deficiencias y normalizar su aprendizaje en el currículo educativo. Así, el apoyo se posiciona como un constructo alejado de ofrecer al centro un recurso común del que pueden beneficiarse todos los agentes educativos (Booth y Ainscow, 2015; Hagiwara et al., 2019).

Otra de las aportaciones de este estudio radica en la escasa flexibilidad que poseen los centros educativos para ofrecer un aprendizaje que llegue a todos. El apoyo se comprende de manera restrictiva y balcanizada, donde los espacios, tiempos y roles docentes estigmatizan e impiden la consecución de un modelo de apoyo inclusivo.

Ello provoca una desigual responsabilidad en las tareas del profesorado hacia la diversidad, ya que es el docente de apoyo el que debe emprender -a veces de manera solitaria- el proceso de inclusión en su centro. Estos hechos se acompañan de una falta de coordinación determinada, en gran medida, por la falta de formación y de tiempo en materia de inclusión del profesorado regular, la carga de trabajo, los roles docentes estigmatizados y las estrategias metodológicas rígidas que acompañan al profesorado.

El apoyo educativo concede a las escuelas la oportunidad o la imposibilidad de crear y asentar una educación verdaderamente inclusiva. Por lo que, siguiendo este principio, el profesorado de apoyo constituye una de las figuras más representativas y proclives a efectuar el cambio, ya que gracias a sus actuaciones, su preparación en materia de diversidad y sus posibilidades de acción dentro de los centros educativos, puede constituirse en el principal referente para acercar la inclusión a las aulas ordinarias, optimizando la atención a la diversidad en todos los rincones de la escuela.

La forma en la que son entendidas las funciones, las tareas y los roles docentes en las escuelas, así como las barreras a las que se enfrenta la inclusión, son cruciales en la creación de apoyos inclusivos, ya que si no se apuesta por una visión conjunta del apoyo, donde toda 
la comunidad educativa participe en su mejora, cualquiera de los esfuerzos bienintencionados de manera aislada se debilitarán en el camino hacia la inclusión.

No se puede acabar este estudio sin mencionar las limitaciones que lo acompañan. En referencia a la muestra, esta investigación no aporta una población significativa -a nivel autonómico- que permita generar resultados extrapolables a otros contextos. Además, la creación de grupos de discusión o la realización de entrevistas hubiera enriquecido los datos obtenidos en el cuestionario.

\section{Referencias}

Ainscow, M. (2017). Haciendo que las escuelas sean más inclusivas: lecciones a partir del análisis de la investigación internacional. Revista de Educación inclusiva, 5(1), 39-49.

Anaby, D. R., Ianni, L., Héguy, L. y Camden, C. (2020). Actual and ideal roles of school staff to support students with special needs: current needs and strategies for improvement. Support for Learning, 35(3), 326-345. doi: 10.1111/1467-9604.12313

Arnaiz, P. (2019). La educación inclusiva en el siglo XXI: avances y desafíos. Lección Magistral Santo Tomás. Murcia: Santo Tomás.

Arnaiz, P. y De Haro, R. (2020). ¿Hacia dónde va la escuela inclusiva? Análisis y necesidades de cambio en centros educativos. En O. Moliner. (Ed.), Acompañar la inclusión escolar (pp. 33-46). Madrid, España: Dykinson, S.L.

Arnaiz, P., Escarbajal, A., Alcaraz, S. y De Haro, R. (2021). Formación del profesorado para la construcción de aulas abiertas a la inclusión. Revista de Educación, 393, 37-67. doi: 10.4438/1988-592X-RE-2021-393-485

Booth, T. y Ainscow, M. (2015). Guía para la educación inclusiva. Desarrollando el aprendizaje y la participación ciudadana en los centros escolares. Bristol, UK: FUHEM, OEI.

Cortés-González, P., González-Alba, B. y Sánchez Mesa, M. de F. (2018). Agrupamientos escolares y retos para la educación inclusiva en infantil y primaria. Tendencias Pedagógicas, 32, 75-90. doi: 10.15366/tp2018.32.006

Dagli, O., Akcamete, G. y Guneyli, A. (2020). Impact of Co-Teaching Approach in Inclusive Education Settings on the Development of Reading Skills. International Journal of Education and Practice, 8(1), 1-17. doi: 10.18488/journal.61.2020.81.1.17

Gómez-Zepeda, G., Petreñas, C., Sabando, D. y Puigdellivol, I. (2017). The role of the Support and Attention to Diversity Teacher (SADT) from a community-based perspective: Promoting educational success and educational inclusion for all. Teaching and Teacher Education, 64, 127-138. doi: 10.1016/j.tate.2017.02.002

Hagiwara, M., Shogren, K. A., Thompson, J. R., Burke, K. M., Uyanik, H., Amor, A. M., ... Aguayo, V. (2019). International trends in inclusive education intervention research: A literature review. Education and Training in Autism and Developmental Disabilities, 54(1), 3-17. Recuperado de: https://search.proquest.com/docview/2183484340?accountid=17225

Härkki, T., Vartiainen, H., Seitamaa-Hakkarainen, P. y Hakkarainen, K. (2021). Co-teaching in non-linear projects: A contextualised model of co-teaching to support educational change. Teaching and Teacher Education, 97, 1-14. doi: 10.1016/j.tate.2020.103188 
Luque, A., Hernández, C. M., Fernández, M. M. y Carrión, J. J. (2018). Docentes de audición y lenguaje itinerante: el trabajo colaborativo, reto pendiente para un nuevo perfil. Revista Educación, 43(1), 170-182. doi: 10.15517/revedu.v43i1.28110

Moya, A. (2012). El profesorado de apoyo en los centros ordinarios. Nuevas funciones, nuevas contradicciones. Educatio Siglo XXI, 30(1), 71-88.

Nadal, M. J., Grau, C. y Peirats, J. (2016). Análisis y valoración del modelo inclusivo en los sistemas de apoyo de centros de infantil y primaria. Educatio Siglo XXI, 34(3), 161-180. doi: $10.6018 / \mathrm{j} / 276001$

Ní Bhroin, Ó. y King, F. (2020). Teacher education for inclusive education: a framework for developing collaboration for the inclusion of students with support plans. European Journal of Teacher Education, 43(1), 38-63. doi: 10.1080/02619768.2019.1691993

Quintero, L. E. (2020). Educación inclusiva: tendencias y perspectivas. Educación y Ciencia, 24, 2-17. doi: 10.19053/0120-7105.eyc.2020.24.e11423

Sandoval, M., Márquez, C., Simón, C. y Echeita, G. (2019). El desempeño profesional del profesorado de apoyo y sus aportaciones al desarrollo de una educación inclusiva. Publicaciones, 49(3), 251-266. doi: 10.30827/publicaciones.v49i2.v49i3.11412

Sandoval, M., Simón, C. y Echeita, G. (2012). Análisis y valoración crítica de las funciones del profesorado de apoyo desde la educación inclusiva. Revista de Educación, número extraordinario, 117-137.

Sanahuja, A., Moliner, O. y Moliner, L. (2020). Organización del aula inclusiva: ¿Cómo diferenciar las estructuras para lograr prácticas educativas más efectivas? Revista Complutense de Educacion, 31(4), 497-506. doi: 10.5209/rced.65774

Sanahuja, J. M., Mas, O. y Olmos, P. (2018). El rol del profesorado de apoyo en el aula regular de un instituto de educación secundaria. Revista Complutense de Educación, 29(4), 1041-1057. doi: 10.5209/RCED.54608

Simón, C., Echeita, G. y Sandoval, M. (2018). Incorporating students' voices in the 'Lesson Study'as a teacher-training and improvement strategy for inclusion. Cultura $y$ Educación, 30(1), 205-225. doi: 10.1080/11356405.2017.1416741

Soldevilla, J., Naranjo, M. y Muntaner, J. J. (2017). Inclusive practices: the role of the support teacher. Aula Abierta, 46, 49-56. doi: 10.17811/rifie.46.2017.49-56

Takala, M., Pirttimaa, R. y Törmänen, M. (2009). Inclusive special education: the role of special education teachers in Finland. British Journal of Special Education, 36(3), 162-173.

Tsvintarnaia, I., Vizcarra, M. T. y López-Vélez, A. L. (2020). Actuaciones llevadas a cabo en la Comunidad Autónoma del País Vasco con alumnado con necesidades específicas de apoyo educativo. REOP-Revista Española de Orientación y Psicopedagogía, 31(1), 81-97. doi: 10.5944/reop.vol.31.num.1.2020.27291 